Universidade Tecnológica Federal do Paraná - UTFPR

Campus Ponta Grossa - Paraná - Brasil

ISSN: 1981-3686 / v. 04, n. 02: p. 243-251, 2010.

D.O.I: $10.3895 / \mathrm{S} 1981-36862010000200012$
Revista Brasileira de Tecnologia

Agroindustrial

\title{
AVALIAÇÃO SENSORIAL E INFLUÊNCIA DO TRATAMENTO TÉRMICO NO TEOR DE ÁCIDO ASCÓRBICO DE SORVETE DE PIMENTA
}

\section{SENSORY EVALUATION AND THE INFLUENCE OF HEAT TREATMENT ON THE ASCORBIC ACID CONTENT OF PEPPER ICE CREAM}

\author{
Flávia Leontina Andrade Dutra ${ }^{1}$, Ivanise Guilherme Branco ${ }^{2}$, \\ Grasiele Scaramal Madrona $^{3}$, Charles Windson Isidoro Haminiuk ${ }^{4}$ \\ ${ }^{1}$ Universidade Estadual de Maringá, Maringá, PR flaviadutra00@hotmail.com \\ ${ }^{2}$ Universidade Estadual Paulista (UNESP), Campus de Assis, SP igbranco@assis.unesp.br \\ ${ }^{3}$ Universidade Estadual de Maringá, Maringá, PR grasiele@yahoo.com \\ ${ }^{4}$ Universidade Tecnológica Federal do Paraná (UTFPR), Campo Mourão, PR haminiuk@utfpr.edu.br
}

\begin{abstract}
Resumo
Neste trabalho foram elaborados três sorvetes adicionados com pimentas de diferentes variedades: dedo-de-moça (Capsicum baccatum), japaleño (Capsicum annuum) e malagueta (Capsicum frutescens). As pimentas foram previamente pasteurizadas e avaliadas em relação ao efeito do tratamento térmico sobre o teor de ácido ascórbico. Resultados mostraram que a pimenta jalapeño apresentou maior teor de ácido ascórbico, porém o tratamento térmico acarretou na maior perda do biocomposto. A maior manutenção do ácido ascórbico foi verificada com a pimenta malagueta, que apresentou teor do biocomposto inferior, porém próximo à pimenta jalapeño. Foi realizada a avaliação da aceitação dos sorvetes através dos atributos sensoriais de cor, aroma, sabor e textura. A análise da variância mostrou que não existe diferença significativa entre as formulações de sorvetes, ao nível de 5\% de significância, em relação à cor, aroma e textura. Em relação ao sabor, as amostras diferiram entre si, ao nível de $5 \%$ de significância, sendo a de maior preferência o sorvete elaborado com pimenta malagueta habanero.
\end{abstract}

Palavras-chave: pimenta, sorvete, ácido ascórbico, tratamento térmico.

\section{INTRODUÇÃ̃}

O crescente mercado dos produtos naturais, aliado ao interesse dos consumidores na prevenção de doenças, tem pressionado a indústria alimentícia na busca por produtos mais saudáveis, direcionando assim pesquisas nesse seguimento. Neste contexto, os alimentos funcionais ganharam prestígio pelos efeitos benéficos que promovem à saúde e, entre esses alimentos, destacam-se as pimentas (ANJO, 2004). 
As pimentas (Capsicum spp.) compõem uma importante parte do mercado de hortaliças frescas do Brasil, e também do segmento de condimentos, temperos e conservas, a nível mundial (COSTA et al., 2008). As pimentas pertencem à família Solanaceae, gênero Capsicum, que apresenta mais de 150 variedades, todas derivadas de cinco espécies cultivadas: $C$. аппиит, $C$. baccatum, C. chinese, C. frutescens e C. pubescens (BONTEMPO, 2007).

A pungência ou picância das pimentas deve-se a presença da capsaicina, substância química que confere à pimenta seu caráter ardido e propriedades benéficas à saúde. A capsaicina apresenta propriedades medicinais comprovadas, atua como cicatrizante, antioxidante, bactericida, auxilia na dissolução de coágulos sanguíneos, previne a arteriosclerose, controla o colesterol, evita hemorragias, aumenta o gasto calórico e influencia na liberação de endorfinas (GREER, 2006; BONTEMPO, 2007).

As pimentas apresentam uma diversidade em sua composição química. Entre os principais componentes destacam-se os capsaicinóides, os carotenóides e o ácido ascórbico, sendo que os níveis de compostos podem variar de acordo com o genótipo (TOPUZ e OZDEMIR, 2007) e grau de maturação (KAPPEL, 2007).

O teor de carotenóides e ácido ascórbico aumenta com a maturação dos frutos de pimenta (HOWARD et al, 1994), sendo também influenciados por condições de processamento, como a pasteurização (HOWARD et al., 1994), a secagem (SCALA e CRAPISTE, 2008), a irradiação (TOPUZ e OZDEMIR, 2003; TOPUZ e OZDEMIR, 2004), entre outros .

Em alimentos, o ácido ascórbico tem função muito importante devido sua ação fortemente redutora. É largamente empregado como agente antioxidante para estabilizar cor, sabor e aroma em alimentos. Além do emprego como conservador, o ácido ascórbico é utilizado para enriquecimento de alimentos ou restauração, a níveis normais, do valor nutricional perdido durante o processamento (ARGANDOÑA, 2005).

Existe uma grande variedade de pimentas, sendo destaque, entre as picantes, as pimentas malagueta e jalapeño. A pimenta malagueta (Capsicum frutescens) é uma das mais utilizadas na culinária e na medicina popular brasileira. Os frutos são pequenos e vermelhos quando maduros e possuem aroma e sabor forte, bastante picante. A pimenta jalapeño (Capsicum anuum) é uma famosa pimenta, cujos frutos são cônicos, de coloração verde-claro a verde-escuro quando não maduros e vermelha quando maduros. Já a pimenta dedo-de-moça (Capsicum baccatum), é uma das mais consumidas no Brasil. Os frutos são alongados e pendentes, com coloração verde (imaturo) e vermelha (maduro), provocando ardência com moderação. Quando seca e moída é conhecida como pimenta calabresa (BONTEMPO, 2007).

Atualmente, há produtos altamente consumidos no mercado como, por exemplo, os sorvetes. De acordo com a Associação Brasileira das Indústrias de Sorvetes (ABIS, 2009), a produção anual 
chega a 900 milhões de litros, sendo que o Brasil ocupa o $10^{\circ}$ lugar no ranking mundial de produção e o $11^{\circ}$ em consumo, com média anual de 4,7 litros de sorvete per capita.

Do ponto de vista nutricional, o sorvete é classificado como excelente fonte de energia e considerado um alimento completo e de alto valor nutritivo, pois fornece proteínas, carboidratos, lipídeos, vitaminas A, B1, B2, B6, C, D, E e K, cálcio, fósforo e outros minerais. Os sorvetes combinam muito bem com o clima tropical do país, existindo variados ingredientes que podem ser usados para enriquecer e diversificar ainda mais os sorvetes, que vão desde as frutas mais exóticas até sementes dos mais diversos tipos (ARBUCKLE, 1986).

Nesse sentido, neste trabalho foi elaborado um sorvete com adição de pimenta, agregando assim valor a um produto bastante consumido e proporcionando ao sorvete propriedades funcionais por meio da incorporação da pimenta, e avaliar a aceitação sensorial do produto obtido. Outro objetivo do presente trabalho foi avaliar a influência do tratamento térmico na perda de ácido ascórbico durante o branqueamento prévio das pimentas. A avaliação das perdas de ácido ascórbico durante o processamento é de extrema importância, visto ser um componente facilmente destruído por calor e luz e sua quantificação após o processamento constituirá um importante fator para o controle de qualidade do produto elaborado.

\section{MATERIAL E MÉTODOS}

\section{Material}

As variedades de pimenta utilizadas foram "Dedo-de-Moça" (Capsicum baccatum), Japaleño (Capsicum annuum) e Malagueta habanero (Capsicum frutescens), adquiridas no comércio local de Maringá (PR).

Para a elaboração do sorvete foram utilizados leite integral (Polly - Confepar), estabilizanteemulsificante e super liga neutra (Selecta - Duas Rodas Industrial Brasil Ltda). Os outros ingredientes adicionados, bem como açúcar e limão utilizados no preparo da calda, também foram adquiridos no comércio local de Maringá (PR).

\section{Métodos}

\section{Processamento do Sorvete e Calda}

O processamento do sorvete foi realizado no laboratório de Engenharia de Alimentos da Universidade Estadual de Maringá (UEM), de acordo com a metodologia proposta no rótulo da embalagem da Selecta, da empresa Duas Rodas Industrial Ltda, diferenciando apenas na adição da pimenta previamente branqueada. Nas formulações dos sorvetes foram adicionados $1,5 \%$ de cada 
variedade de pimenta, que foram "Dedo-de-Moça" (Capsicum baccatum), Japaleño (Capsicum annuит) e Malagueta (Capsicum frutescens).

As pimentas utilizadas para o processamento do sorvete foram previamente selecionadas, lavadas com água clorada $(25 \mathrm{ppm})$ e branqueadas em água fervente $\left(100^{\circ} \mathrm{C}\right)$ por três minutos, seguindo de imersão em água fria.

Para a calda, foram homogeneizados em um liquidificador doméstico a pimenta, o suco de limão, água e açúcar, na proporção de 1:1:3:4 respectivamente, e levados para concentração, sob temperatura $100^{\circ} \mathrm{C}$, até atingir $65^{\circ} \mathrm{Brix}$.

Foram elaboradas três formulações de sorvete e, conseqüentemente, de calda alterando a variedade de pimenta.

\section{Análises físico-químicas}

As análises de $\mathrm{pH}$, acidez total titulável, umidade e cinzas foram realizadas nos sorvetes adicionados com pimenta branqueada, de acordo com as Normas Analíticas do Instituto Adolfo Lutz (IAL, 1985).

A determinação de ácido ascórbico foi realizada nas pimentas antes e após o branqueamento, utilizando a metodologia proposta por Benassi e Antunes (1998).

\section{Análise Sensorial}

A análise sensorial das formulações foi feita pela avaliação da aceitação através da escala hedônica de 1 (desgostei muito) a 7 (gostei muito) pontos, para avaliação de cor, aroma, sabor e textura. Uma equipe de 50 provadores não treinados, ambos os sexos, avaliou as três formulações. As amostras foram codificadas com números de três dígitos e a ordem de apresentação foi aleatorizada entre os provadores. A análise estatística dos resultados foi realizada utilizando-se análise de variância (ANOVA) e cálculo de médias por Tukey, teste que deve ser aplicado toda vez que se pretende comparar as médias dos tratamentos.

\section{RESULTADOS E DISCUSSÃO}

\section{Caracterização físico-química do sorvete com pimenta}

Os resultados médios obtidos das determinações físico-químicas dos sorvetes, adicionados de pimentas previamente branqueadas, estão apresentados na Tabela 1.

Tabela 1. Caracterização físico-químicas dos sorvetes de pimenta

\begin{tabular}{ccccc}
\hline Variedades & $\mathbf{p H}$ & Acidez (\%) & Umidade (\%) & Cinzas (\%) \\
\hline Capsicum baccatum & 6,59 & 0,022 & 63 & 0,50 \\
Capsicum annuum & 6,72 & 0,023 & 68 & 0,82 \\
Capsicum frutescens & 6,60 & 0,026 & 67 & 0,23 \\
\hline
\end{tabular}


Comparando os resultados da Tabela 1, observa-se que o teor de umidade e cinzas estão próximos aos obtidos por SANTANA et al. (2003), caracterizando sorvetes elaborados com diferentes genótipos de mamão, cuja faixa umidade encontrada foi de 66 a $69 \%$ e cinzas entre 0,33 a $0,51 \%$. No entanto, os resultados de $\mathrm{pH}(5,53$ a 5,78) e acidez total titulável $(0,11$ a 0,13$)$ estão ligeiramente fora da faixa quando comparados com o presente trabalho.

Os resultados médios de ácido ascórbico, das diferentes variedades de pimenta, antes e após o tratamento térmico são apresentados na Tabela 2.

Tabela 2. Teor de ácido ascórbico das pimentas antes e após branqueamento

\begin{tabular}{lccc}
\hline \multicolumn{1}{c}{ Variedades } & $\begin{array}{c}\text { Antes do branqueamento } \\
\text { (mg/100 g amostra) }\end{array}$ & $\begin{array}{c}\text { Após o branqueamento } \\
\text { (mg/100 g amostra) }\end{array}$ & $\begin{array}{c}\text { \% Redução de } \\
\text { ácido ascórbico }\end{array}$ \\
\hline Capsicum baccatum & 160,00 & 115,23 & 27,00 \\
Capsicum annuum & 238,94 & 159,30 & 33,33 \\
Capsicum frutescens & 215,48 & 176,30 & 18,18 \\
\hline
\end{tabular}

Verifica-se na Tabela 2 que o teor de ácido ascórbico das pimentas in natura estudadas foram diferentes, de acordo com a variedade analisada, podendo ser devido às variações nos cultivares, genética, maturidade, fertilização e condições ambientais (SIMMONE et al., 1997). Entretanto, o teor de ácido ascórbico, quando comparado com a literatura, está dentro do intervalo teórico encontrado em outros estudos. HOWARD et al. (1994) obtiveram teor de ácido ascórbico entre 75-277 mg /100 g de amostra enquanto CASTRO et al. (2008) verificaram 107,4 mg de ácido ascórbico/100 g de amostra.

No estudo do efeito do branqueamento sobre a estabilidade do ácido ascórbico durante o processamento das pimentas, observou-se redução significativa deste biocomposto, variando entre 18 a 34\% em relação ao valor inicial encontrado. Essa perda pode ser devido à lixiviação de compostos antioxidantes (CHUAH et al., 2008), além do tratamento térmico aplicado, que acelera a oxidação do ácido ascórbico para ácido dehidroascórbico, seguido pela hidrólise a ácido 2,3dicetogulônico e para outros componentes nutricionalmente inativos (GREGORY, 1996). Assim, para melhor aproveitamento de compostos bioativos presentes nos pigmentos, é imprescindível utilizar menor quantidade de água no branqueamento e reduzir o tempo de cozimento, além de aproveitar a água utilizada no tratamento térmico (CHUAH et al, 2008).

A variedade Capsicum annuum obteve a maior perda de ácido ascórbico, enquanto que a menor foi verificada com a Capsicum frutescens. Assim, sorvetes elaborados com esta última variedade de pimenta mantém maior quantidade deste biocomposto de interesse.

\section{Análise Sensorial}

A Tabela 3 apresenta os resultados obtidos na avaliação sensorial para os atributos cor, aroma, sabor e textura dos sorvetes, com suas respectivas caldas, elaborados com as três variedades de pimenta. 
Tabela 3. Valores médios das notas atribuídas pelos provadores para as características sensoriais dos sorvetes e

\begin{tabular}{ccccc}
\multicolumn{5}{c}{ caldas de pimenta. } \\
\hline Variedades & Cor & Aroma & Sabor & Textura \\
\hline Capsicum baccatum & $6,88^{\mathrm{a}}$ & $5,09^{\mathrm{a}}$ & $5,20^{\mathrm{a}}$ & $5,97^{\mathrm{a}}$ \\
Capsicum annuum & $6,40^{\mathrm{a}}$ & $5,03^{\mathrm{a}}$ & $4,97^{\mathrm{b}}$ & $5,71^{\mathrm{a}^{\mathrm{a}}}$ \\
Capsicum frutescens & $6,60^{\mathrm{a}}$ & $5,03^{\mathrm{a}}$ & $6,29^{\mathrm{c}}$ & $6,23=$ \\
\hline
\end{tabular}

Médias com letras iguais numa mesma coluna não diferem estatisticamente entre si $(p>0,05)$.

Em relação à cor, os sorvetes alcançaram médias correspondentes aos termos da escala hedônica "gostei muito", mostrando uma elevada aceitação dos produtos. Verifica-se na Tabela 3 que não houve diferença significativa $(p>0,05)$ em relação ao atributo cor, independente da variedade de pimenta utilizada na formulação. Este resultado é particularmente interessante, pois as diferenças verificadas na aparência das pimentas in natura, característica de cada variedade, desapareceram no produto processado. Assim, independente da variedade utilizada na formulação, as amostras de sorvete de pimenta obtiveram uma grande aceitação para o atributo sensorial cor.

Como verificado para atributo cor, o aroma também não apresentou diferença significativa entre as amostras, correspondendo ao termo da escala hedônica "gostei ligeiramente". Este resultado pode estar relacionado pela presença, mesmo depois do processamento, do aroma característico da pimenta.

Quanto ao sabor, observa-se por meio da Tabela 3, que existe diferença significativa $(\mathrm{p} \leq 0,05)$ entre os sorvetes elaborados com as diferentes variedades de pimenta. Os provadores preferiram o sabor do sorvete, com sua respectiva calda, da espécie Capsicum frutescens, mais amplamente conhecida como habanero. Esta diferença entre as amostras deve-se ao fato das pimentas apresentarem grau de pungência distintos entre si. Entretanto, observa-se que todos os sorvetes e caldas de pimenta apresentaram satisfatória aceitação sensorial com relação a este atributo, correspondendo aos termos da escala hedônica entre "gostei ligeiramente" e "gostei regularmente".

Para o atributo textura, as médias alcançaram aos termos da escala hedônica "gostei ligeiramente" e não apresentaram diferença significativa $(p \leq 0,05)$ entre si. Estes valores mostraram uma boa aceitação dos produtos quanto a esse atributo, embora as variedades de pimentas in natura adicionadas apresentassem ligeira diferença em relação ao grau de maturação.

\section{CONCLUSÃO}

A partir dos resultados obtidos nos experimentos realizados nesse estudo, pode-se concluir que a menor perda de ácido ascórbico com o branqueamento foi com pimenta da variedade Capsicum frutescens. 
Os sorvetes e as caldas, das três diferentes variedades de pimenta, demonstraram boa aceitação para todos os atributos avaliados. A maior aceitação dos provadores, para o atributo sabor, foi o sorvete e a calda preparados com pimenta da espécie Capsicum frutescens. Não houve prevalência entre os produtos quanto à cor, aroma e textura indicando que os diferentes tipos de pimenta poderão ser aproveitados na indústria para elaboração de sorvete.

O sorvete e a calda são, portanto, uma excelente alternativa para o aproveitamento das propriedades funcionais da pimenta.

\begin{abstract}
In this work were prepared with three ice cream added peppers of different varieties: Capsicum baccatum, Capsicum annuum and Capsicum frutescens. The peppers were first pasteurized and evaluated in relation to the effect of heat treatment on the ascorbic acid content. Results showed that the Capsicum annuum showed higher ascorbic acid content, but the heat treatment resulted in greater loss of biocompounds. The higher retention of ascorbic acid was observed with Capsicum frutescens, which presented lower content of biocompound, but near of the pepper Capsicum annuum. The follow attributes was performed to evaluate the acceptance of ice cream through the sensory attributes: color, aroma, flavor and texture. Analysis of variance showed no significant difference between ice cream formulations at the 5\% level of significance with regard to color, aroma and texture. Regarding taste, the samples differed significantly, at 5\% significance, and the most preferred was the ice cream made with pepper $\underline{\text { Capsicum frutescens. }}$
\end{abstract}

Key-words: pepper, ice-cream, ascorbic acid, heat treatment.

\title{
Referências
}

ANJO, D. F. C. Alimentos funcionais em angiologia e cirurgia vascular. Jornal Vascular Brasileiro, v. 3, n. 2. p. 145$154,2004$.

ARBUCKLE, W. S. Creme de gelo. 4. ed. AVI Publishing Companhia, Inc., Westport, Connecticut, 1986.

ARGANDOÑA, S.J.E. Goiabas desidratadas osmoticamente e secas: Avaliação de um sistema osmótico semicontínuo, da secagem e da qualidade. Campinas, 157 f. 2005, Unicamp. Tese de Doutorado. 2005.

(ABIS) ASSOCIAÇÃO BRASILEIRA DAS INDÚSTRIAS DE SORVETE. Jornal de Itatiba - SP Disponível em www.abis.com.br. Acesso em 17 abr. 2009.

BENASSI, M. T.; ANTUNES, A. J. A. Comparison of meta-phosphoric and oxalic acids as extractant solutions for determination of vitamin $C$ in selected vegetables. Arquivos de Biologia e Tecnologia, v.31, n. 4, p. 507-513, 1998. BONTEMPO, M. Pimenta e seus benefícios à saúde - Marcio Bontempo. São Paulo: Alaúde Editorial, 2007.

CASTRO, S. M.; SARAIVA, J. A.; LOPES-DA SIlVA, J. A.; DELGADILlO, I.; LOEY, A. V.; SMOUT, C.; HENDRICKX, M. E. Effect of thermal blanching and of high pressure treatments on sweet green and red bell pepper fruits (Capsicum annuum L.). Food Chemistry, v. 107, n. 4, p. 1436 - 1449, 2008.

CHUAH, A. M; LEE, YA-CHI; YAMAGUCHI, T.; TAKAMURA, H.; YIN, LI-JUN; MATOBA, T. Effect of cooking on the antioxidant properties of coloured peppers. Food Chemistry, v. 111, n. 01, p. 20-28, 2008. DOI:10.1016/j.foodchem.2008.03.022.

COSTA, L.V.; LOPES, M.T.G.; LOPES, R.; ALVES, S.R.M. Polinização e fixação de frutos em Capsicum chinense . Acta Amazonica, v. 38, n. 2, 2008. DOI: 10.1590/S0044-59672008000200022. 
DI SCALA, K.; CRAPISTE, G. Drying kinetics and quality changes during drying of red pepper. Food Science and Technology, v. 41, p. 789-795, 2008.

GREER, L. Pimentos \& Companhia. Lisboa: Lisma - Edição e Distribuição de Livros Ltda, 2006.

GREGORY, J. F. Vitamins. In O. R. Fennema (Ed.). Food Chemistry. 3 ed. New York: Dekker, 1996. p. 531-616.

HOWARD, L. R., SMITH, R. T., WAGNER, A. B., VILlALON, B.; BURNS, E. E. Provitamin A and ascorbic acid content of fresh pepper cultivars (Capsicum annuum) and processed Jalapeños. Journal of Food Science, v. 59, n. 02, p. 362-365, 1994. DOI: 10.1111/j.1365-2621.1994.tb06967.x.

INSTITUTO ADOLFO LUTZ. Normas analíticas - métodos químicos e físicos de análises de alimentos. 3 ed. São Paulo, 1985.

KAPPEL, V. D. Avaliação das propriedades antioxidante e antimicrobiana de extratos de Capsicum baccatum L. var. pendulum. Dissertação (Pós-Graduação em Ciências Biológicas: Bioquímica), Instituto de Ciências Básicas da Saúde, Universidade Federal do Rio Grande do Sul (UFRGS), 2007.

SANTANA, L. R. R.; MATSUURA, F. C. A. U.; CARDOSO, R. L. Genótipos melhorados de mamão (Carica papaya L.): avaliação tecnológica dos frutos na forma de sorvete. Ciência e Tecnologia de Alimentos, v. 23, supl., p. 151-155, 2003.

SIMMONE, A. H.; SIMMONE, E. H.; EITENMILlER, R. R.; MILLS, H. A.; GREEN, N. R. Ascorbic acid and provitamin A contents in some unusually coloured bell peppers. Journal of Food Composition and Analysis. v 10,n. 04, p. 299-311, 1997. DOI: 10.1006/jfca.1997.0544.

TOPUZ, A.; OZDEMIR, F. Influences of g-irradiation and storage on the carotenoids of sun-dried and dehydrated paprika. Journal of Agricultural and Food Chemistry, v. 51, n. 17, p. 4972-4977, 2003. DOI: 10.1021/jf034177z.

TOPUZ, A.; OZDEMIR, F. Influences of gamma irradiation and storage on the capsaicinoids of sun-dried and dehydrated paprika. Food Chemistry, v. 86, n. 04, p. 509-515, 2004. DOI: 10.1016/j.foodchem.2003.09.003.

TOPUZ, A; OZDEMIR, F. Assessment of carotenoids, capsaicinoids and ascorbic acid composition of some selected pepper cultivars (Capsicum annuиm L.) grown in Turkey. Journal of Food Composition and Analysis, v. 20, n. 7, p. 596-602, 2007. DOI: 10.1016/j.jfca.2007.03.007.

YAMASHITA, F.; BENASSI, M. T. Influência da embalagem de atmosfera modificada e do tratamento com cálcio na cinética de degradação de ácido ascórbico e perda de massa em goiabas (Psidium guajava L.). Ciência e Tecnologia de Alimentos, v.20, n. 1, p.27-31, 2000. DOI: 10.1590/S0101-20612000000100006.

\section{Dados dos autores:}

Nome completo: Ivanise Guilherme Branco

Filiação institucional: Universidade Estadual Paulista (Unesp), Câmpus de Assis.

Departamento: Ciências Biológicas

Função ou cargo ocupado: Professor Assistente Doutor

Titulação: Doutorado

Endereço: Av. Dom Antônio, 2100, Cep 19806-900, Assis/SP.

Telefones para contato: (18) 33025848 (18) 97444302

e-mail: igbranco@assis.unesp.br

Nome completo: Flávia Leontina Andrade Dutra

Filiação institucional: Universidade Estadual de Maringá

Departamento: Engenharia Química; Curso Engenharia de Alimentos

Função ou cargo ocupado: Engenheira de Alimentos

Titulação: Graduação

Endereço: R. Prefeito Humberto Pavanelli, 426. Centro. Bela Vista do Paraíso/PR 
Telefones para contato: (43) 3242-1552

e-mail: flaviadutra_00@hotmail.com

Nome completo: Grasiele Scaramal Madrona

Filiação institucional:Universidade Estadual de Maringá (UEM)

Departamento: Engenharia Química; Curso Engenharia de Alimentos

Função ou cargo ocupado: Professor Assistente

Titulação: Mestre

Endereço: Av. Colombo, n 5790, Bloco D-90, Engenharia Química, Cep 87020-900, Maringá/PR.

Telefones para contato: (44) 32614755

e-mail: grasiele@yahoo.com

Nome completo: Charles Windson Isidoro Haminiuk

Filiação institucional: Universidade Tecnológica Federal do Paraná

Departamento: Engenharia de Alimentos

Função ou cargo ocupado: Professor Doutor

Titulação: Doutor

Endereço: BR 369, Km 0,5, Cep 87301-005, Campo Mourão/ PR

Telefones para contato: (44) 35234156

e-mail: haminiuk@utfpr.edu.br 